International Journal of Current Advanced Research

ISSN: O: 2319-6475, ISSN: P: 2319 - 6505, Impact Factor: SJIF: 5.995

Available Online at www.journalijcar.org

Volume 6; Issue 3; March 2017; Page No. 2713-2716

DOI: http://dx.doi.org/10.24327/ijcar.2017.2716.0085

Research Article

\title{
ASSESSMENT OF THE BRUSHING HABITS IN CHILDREN BELOW 6 YEARS - A SURVEY IN SEMI URBAN AREAS
}

\author{
Pranati .T*., Dhanraj M and Marian AnandBennis
}

Department of Prosthodontics, Saveetha Dental College \& Hospitals, Chennai - 600077

\begin{tabular}{l}
\hline A R T I C L E I N F O \\
\hline Article History: \\
Received $10^{\text {th }}$ December, 2016 \\
Received in revised form $16^{\text {th }}$ January, 2017 \\
Accepted $4^{\text {th }}$ February, 2017 \\
Published online $28^{\text {th }}$ March, 2017
\end{tabular}

Key words:

Brushing habits, Brushing habits in children, Brushing habits in semi urban areas

\begin{abstract}
A B S T R A C T
Aim: To do a survey on the brushing habits of children below 6 years in semi urban areas. Objective: This study investigates the brushing habits in children below 6 years living in semi urban areas. It also shows the percentage of people affected with caries.

Background: Brushing teeth is an important part of dental care routine. Improper brushing habits results in the formation of plaque which eventually leads to the formation of cavities. Proper brushing habits should be taught to children for maintaining their oral hygiene and to prevent cavities. This study shows the amount of exposure about the benefits of proper brushing among parents and children belonging to semi urban areas.

Methods: A survey was conducted among randomly selected 118 school children, in a semi urban area (Chittoor district) whose age is below six years. The questionnaire was given to parents as children would not be able to understand or respond to it.

Reasons: One of the major causes for the occurrence of cavities in children is poor brushing habits. This study is to create awareness among parents about the importance of proper brushing habits and to train their children to develop good oral hygiene.

Results: Among 118 children, 82 brushed their teeth only once a day i.e. 69.49\%.Majority didn't use other cleaning materials like mouthwash, interdental brush and floss ( 96 children i.e. $81.36 \%$ ), 21 children i.e. $17.79 \%$ used mouthwash and 1 girl uses floss i.e. $0.84 \%$.Only 20 children $(16.95 \%)$ visit dentist regularly, 80 children don't visit $(67.79 \%)$ and 18 children $(15.25 \%)$ visited dentist at times but not regularly.

Conclusion: This study exposes that there is a lacunae in the awareness of oral hygiene but not to alarming levels.However further dental awareness measures needs to be initiated to address this issue.
\end{abstract}

Copyright $\bigcirc 2017$ Pranati .T et al. This is an open access article distributed under the Creative Commons Attribution License, which permits unrestricted use, distribution, and reproduction in any medium, provided the original work is properly cited.

\section{INTRODUCTION}

Brushing teeth is an important part of dental care routine. Among oral diseases, dental caries is a prevalent dental problem among children [1]. Improper brushing habits results in the formation of plaque which eventually leads to the formation of cavities [2]. Proper brushing habits should be taught to children for maintaining proper oral hygiene and to prevent cavities $[3,4]$. People living in developing countries, and concomitantly, of lower socio- economic status have a lacunae in oral health awareness mirrored in their practice of oral hygiene habits [5]. This study was done to explore the amount of exposure about the benefits of proper brushing habits among parents and children belonging to semi urban areas.

\section{MATERIALS AND METHODS}

This study was carried among randomly selected 118 school

*Corresponding author: Pranati .T

Department of Prosthodontics, Saveetha Dental College \&

Hospitals, Chennai - 600077 children, in a semi urban area (Chittoor district) whose age is below six years. The questionnaire was given to parents as children would not be able to understand or respond to it. Permission was obtained from the school'sheadmistress to conduct the survey. This survey was done after obtaining parents consent. The questionnaire involved around 15 questions from which the brushing habits of children was assessed.

\section{RESULTS}

The responses from 118 parents were collected and the data was analysed and has been tabulated along with charts for each question. Among 118 children, 82 brushed their teeth only once a day i.e. $69.49 \%$ and 36 children i.e. $30.51 \%$ brushed their teeth twice a day (graph 1). Majority use toothbrush and toothpaste for cleaning their teeth (106 children i.e. $89.83 \%$ ), 11 children i.e. $9.32 \%$ use finger and $0.85 \%$ i.e. 1 boy uses tooth powder for cleaning his teeth (graph 2). 


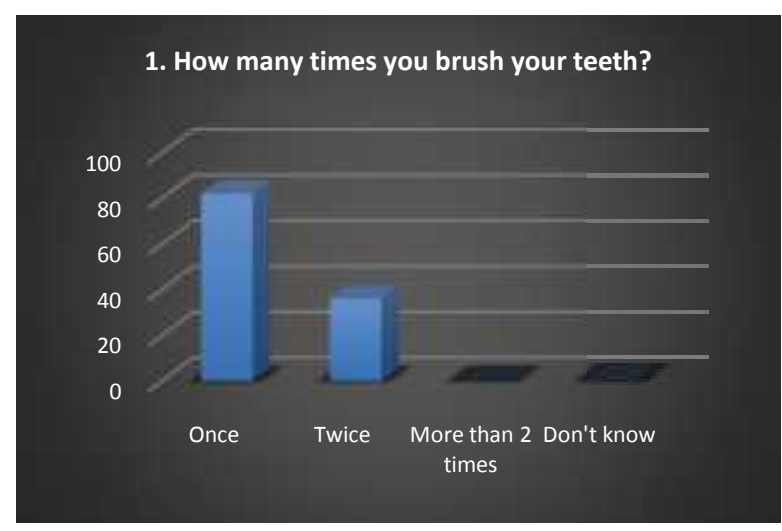

2. What do you use for cleaning your teeth?

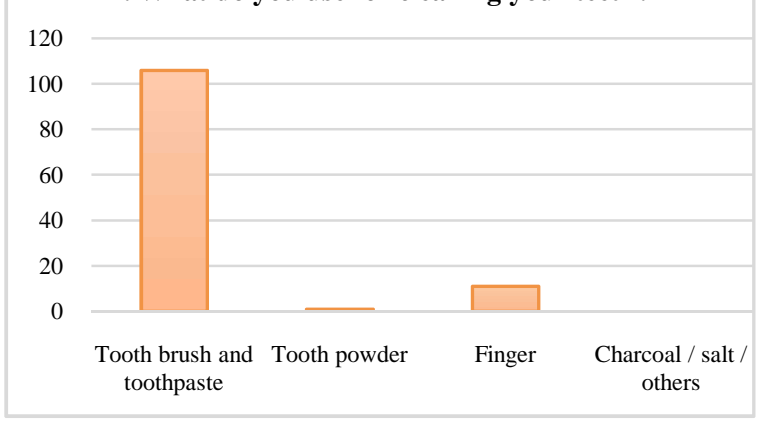

When asked about the brushing techniques, 59 children $(50 \%)$ brushed horizontally, $10.17 \%$ i.e. 12 children used vertical method for brushing, 29 children $(24.5 \%)$ use round or rotatory technique and $15.25 \%$ i.e. 18 children used random technique for brushing their teeth (graph 3).

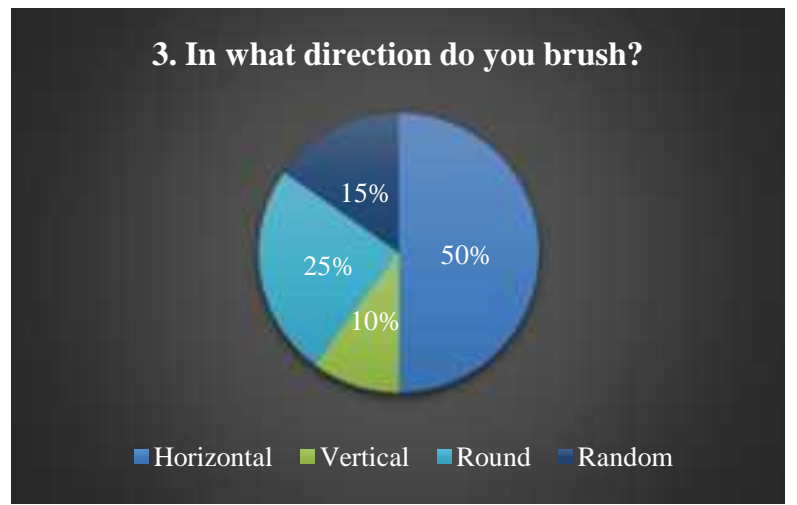

$54.24 \%$ i.e. 64 children brushed their teeth for 1-2 minutes, 32 children $(27.11 \%)$ had brushing frequency greater than 2 minutes and 22 children (18.64\%) brushed for 30 seconds (graph 4).

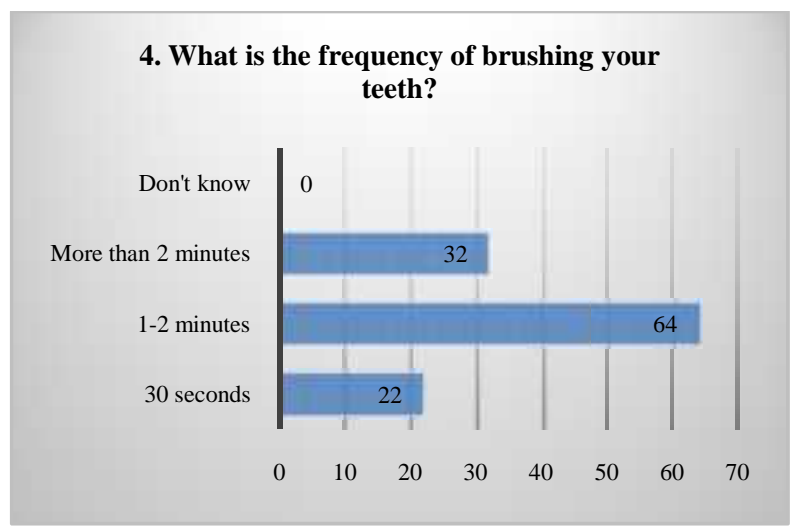

21 kids (17.79\%) changed their brush every month, 44 children $(37.28 \%)$ changed their brush once in 3 months and 42 children $(35.59 \%)$ changed their brush once in 6 months (graph 5)

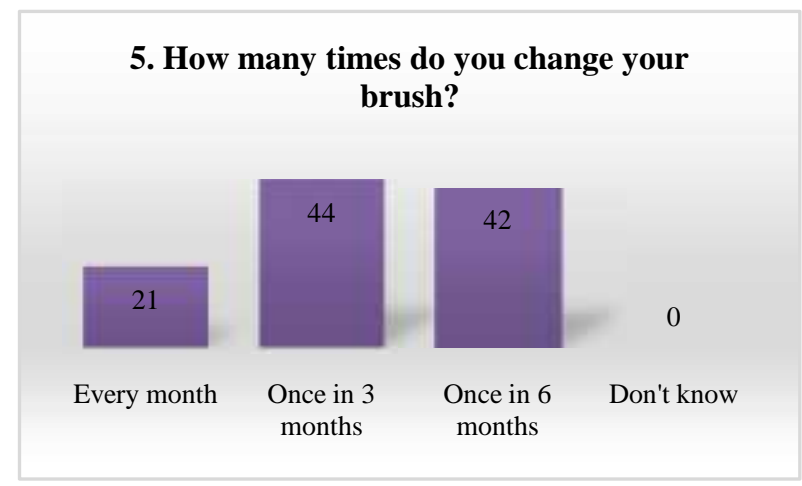

Majority used pea sized amount of toothpaste (60 children i.e. $50.85 \%), 18$ children i.e. $15.25 \%$ used rice grain size and 37 children i.e. $31.35 \%$ used more toothpaste i.e. more than pea sized (graph 6).

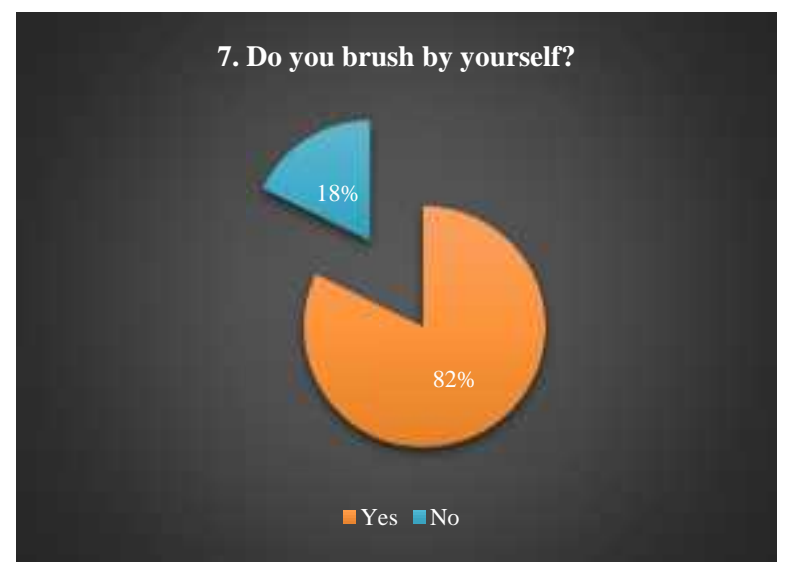

97 children $(82.2 \%)$ brushed by themselves andthe rest i.e. 21 children (17.79\%) were assisted by their parents (graph 7).

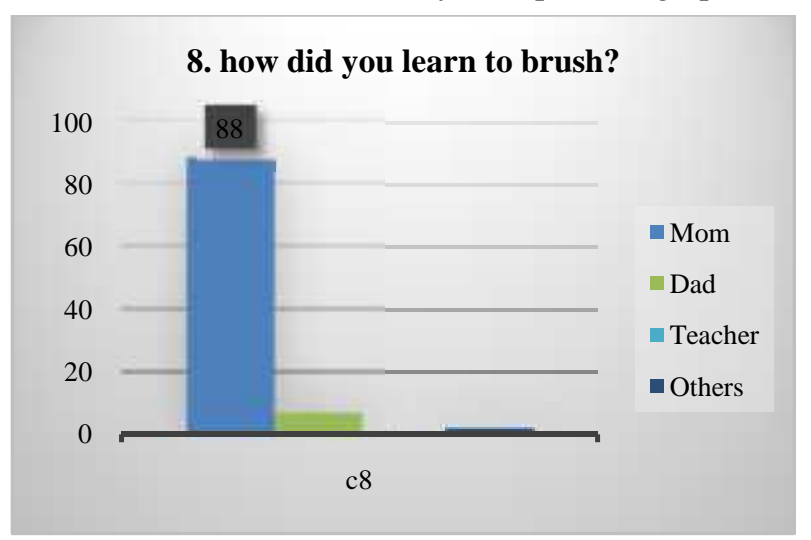

Among the 97 children who brush by themselves, 88 children $(90.72 \%)$ were taught to brush by their mother, 7 children $(7.22 \%)$ by their father and 2 children $(2.06 \%)$ by others (graph 8). 87 children $(89.69 \%$ ) learnt to brush when they were 2-3 years old, 7 children $(7.22 \%)$ at the age of 3-4 years and 3 children (3.09\%) at the age of 4-5 years (graph 9). 50\% of the responses i.e. 59 children said that they cleaned their tongue and the rest did not (graph 10). Majority didn't use other cleaning materials like mouthwash, interdental brush and floss (96 children i.e. 81.36\%), 21 children i.e. $17.79 \%$ used mouthwash and 1 girl uses floss i.e. $0.84 \%$ (graph 11). 

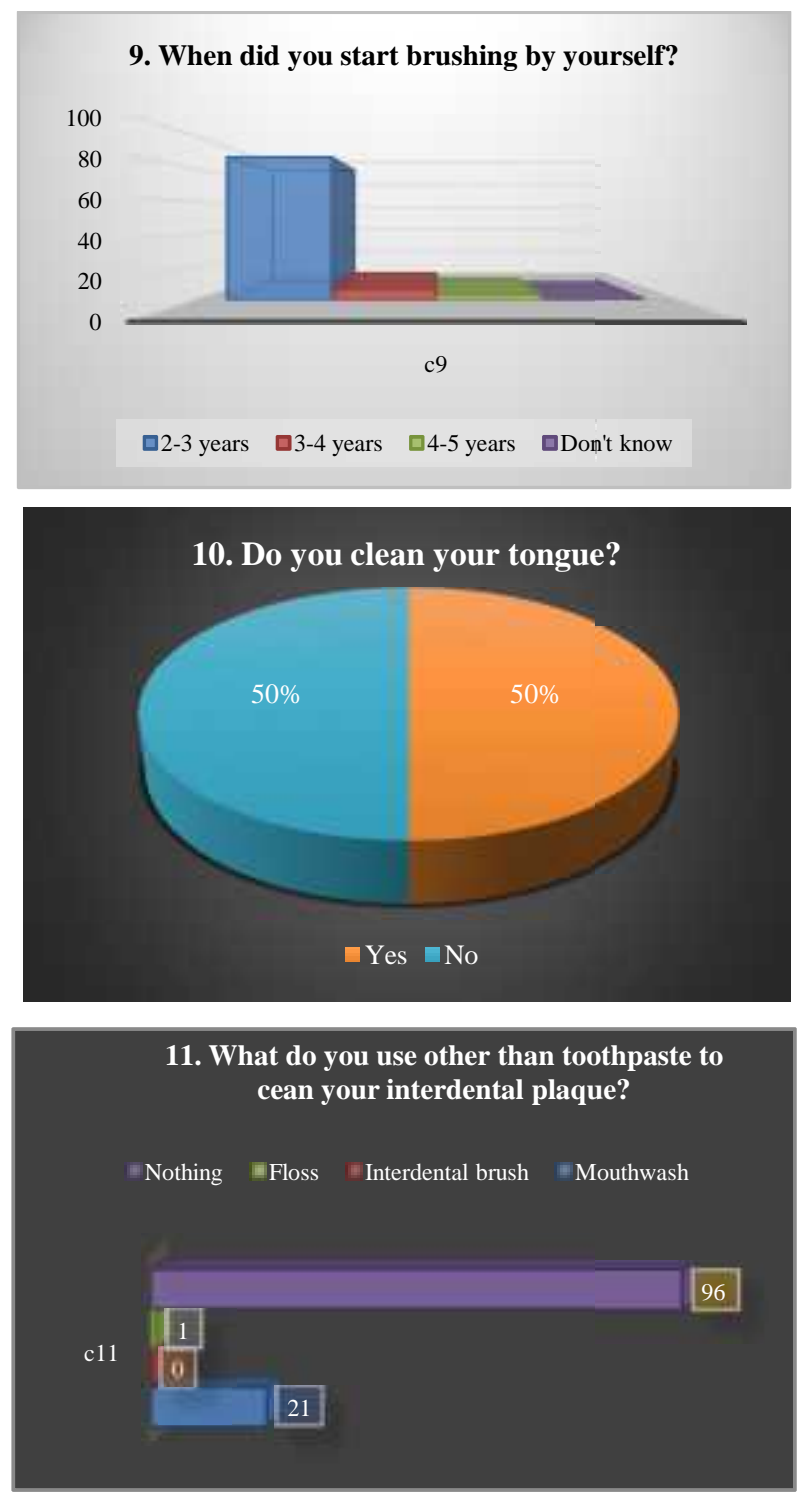

36 children i.e. $30.5 \%$ use soft bristled brush and the rest 71 children $(60.17 \%)$ use medium grade bristled brush (graph 12).

\section{Which toothbrush do you use?}

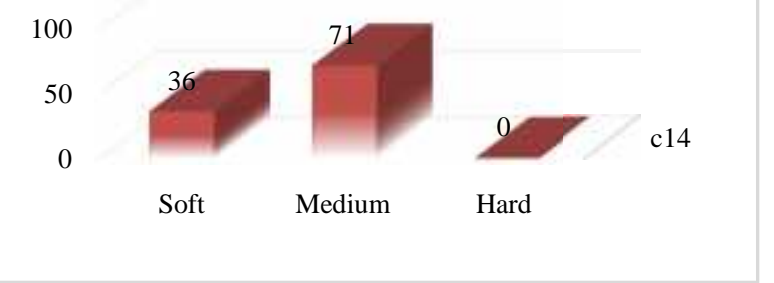

Only 20 children $(16.95 \%)$ the dentist regularly, 80 children don't visit (67.79\%) and 18 children $(15.25 \%)$ visited dentist at times but not regularly (graph 13). Among the 38 children who visit dentist, 24 children visited 3 months ago (20.33\%), 12 children visited a year ago $(10.17 \%)$ and 2 children visited long back (more than a year ago) (graph 14). 77 children $(65.25 \%)$ had no caries and the remaining 41 children $(34.74 \%)$ had caries (graph 15).

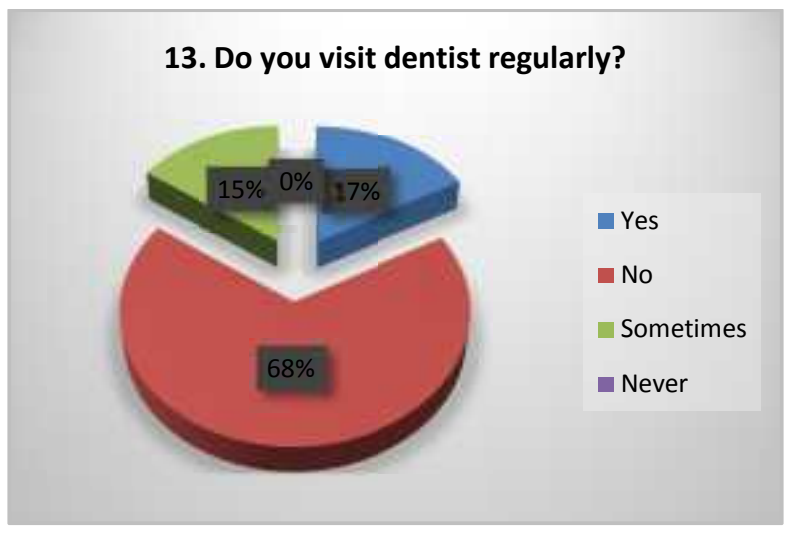

14. When was the last time you visited dentist?
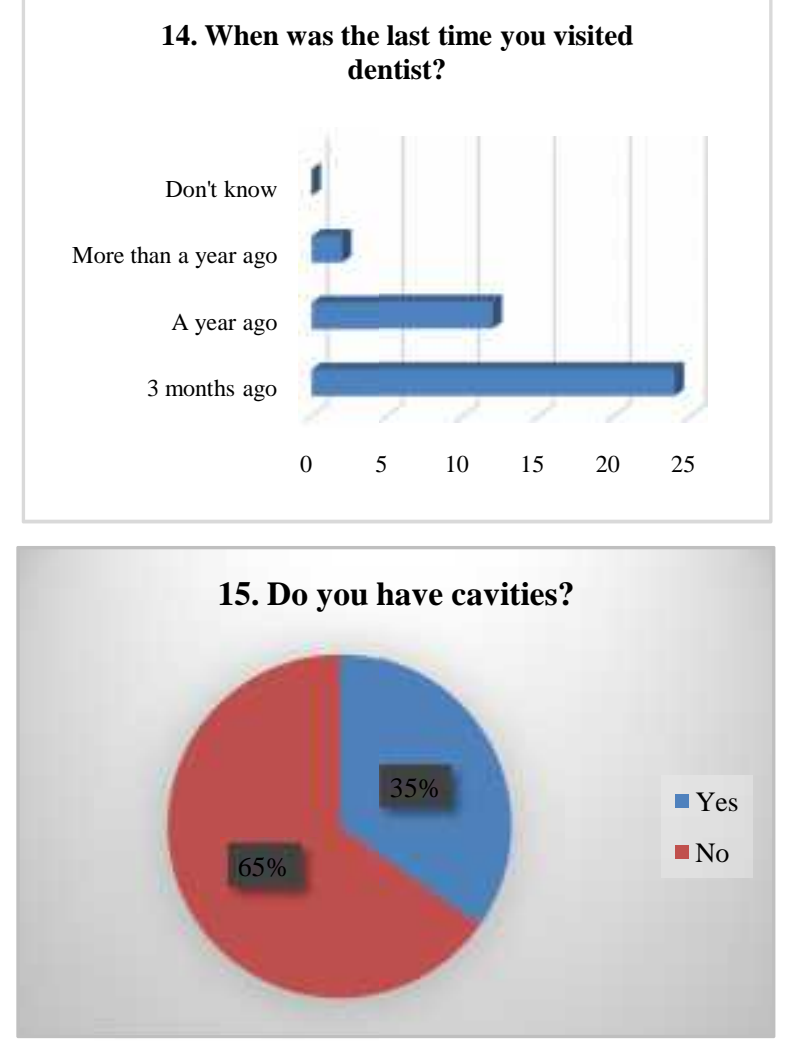

\section{DISCUSSION}

This study shows that only $30.51 \%$ brushed their teeth twice a day i.e. there is lack of awareness among the public (in semi urban areas) about the importance of brushing twice a day.This is in contrast with a similar study by Mohammed Ahad et al [5] among children aged between 12-17 in Chennai, where $53.20 \%$ brushed heir teeth twice a day. The results were similar to a study done by ST Prashanth et al [6] where only $37.65 \%$ children answered that they brushed twice a day.. Majority of them $(89.83 \%)$ use toothbrush and toothpaste for cleaning their teeth. This shows that people have stopped following old, traditional way to clean teeth using charcoal, salt etc. Only 18 children i.e. $15.25 \%$ used random strokes for brushing and the remaining 100 children $(84.75 \%)$ are aware of brushing techniques and they followed certain direction to brush. Among the 100 children majority (59 children) used horizontal strokes to brush. When it comes to brushing frequency, more awareness is required wherein only half i.e. $54.24 \%$ (closer to half) brushed their teeth for 12 minutes. Majority (50.85\%) used pea sized amount of toothpaste i.e. the optimal amount of toothpaste.Majority 
children $(81.36 \%)$ do not practice interdental cleaning habits which is again in contrast with the study done by JayakumariMuttappallymyalil et al in Kerala where $58 \%$ practiced interdental cleaning habits [7] In a study done by Navneet Grewal on 100 Indian children, only 35\% never visited dentist [8] but in this study only $32.2 \%$ visited dentist which is similar to the study done by Zhu L P et al $35.3 \% 12$ years and 20.2\% 18 years visited dentist [9] A positive result that was brought out was $34.74 \%$ had caries which is in total contrast with the study done by Meenu Mittal et al in Gurgaon on 5 year aged children wherein $68.5 \%$ had dental caries [10].

\section{CONCLUSION}

This study exposes that there is a lacunae in the awareness of oral hygiene but not to alarming levels. There is still a need for awareness initiative for oral health at the grassroots level in primary educational institutions with the co-operation and implementation of good oral health practices.A little extra care by the parent or caretaker regarding oral hygiene can give drastic results in reduction of dental caries. In children, the occurrence of periodontal diseases is minimal. Hence, this study focused only on the brushing habits and prevalence of caries.

\section{References}

1. Saravanan S, Kalyani V, Vijayarani MP, Jayakodi P, Felix J, Arunmozhi P, et al. Caries prevalence and treatment needs of rural school children in ChidambaramTaluk, Tamil Nadu, South India. Indian J Dent Res. 2008; 19:186-90. [PubMed: 18797092]

2. Habibian, Mina, et al. "Relationships between dietary behaviours, oral hygiene and mutans streptococci in dental plaque of a group of infants in southern England." Archives of oral biology 47.6 (2002): 491498.
3. Cleaning your teeth and gum. Available www.ADA.org/public/topic/cleaning.asp. [Accessed June 23,2009].

4. J.wood groove,G. Cumber Batch, and S. Gylbier," Understanding dental attendance behavior", community dental health 1987;4:215-21

5. Mohammed Ahad, Gheena.S " Awareness of tooth brushing techniques and proper oral hygiene among school children", J. Pharm. Sci. \& Res. Vol. 7(6), 2015, 367-372

6. PrashanthST, Bhatnagar S, DasUM, Gopu H.Oral health knowledge, practice, oral hygiene status, and dental caries prevalence among visually impaired children in banglore. Journal of the Indian society of pedodontics and preventive dentistry. 2011 Aprjun;29(2):102-5

7. Muttappallymyalil, Jayakumary, et al. "Oral health behaviour among adolescents in Kerala, India." Italian Journal of Public Health 6.3 (2012).

8. GrewalN, Kaur M. Status of oral health awareness in Indian children as compared to western children:A thought provoking situation( A pilot study). J Indian socpedodprev dent 2007;25:15-9

9. Zhu LP, P. E. Wang, H. Y. Bian, J. Y. Zhang, B. X.. Oral health knowledge, attitudes and behaviour of children and adolescents in China. Int Dent J. 2003 Oct;53(5):289-98.

10. Mittal, Meenu, et al. "Oral health status of 5 years and 12 years old school going children in rural Gurgaon, India: an epidemiological study." Journal of Indian Society of Pedodontics and Preventive Dentistry 32.1 (2014): 3 .

\section{Please cite this article in press as:}

Pranati .T et al (2017), Assessment of the Brushing Habits In Children Below 6 years - a Survey In Semi Urban Areas, International Journal of Current Advanced Research, 6(3), pp. 2713-2716.

http://dx.doi.org/10.24327/ijcar.2017. 2716.0085 